Journal of Applied Fluid Mechanics, Vol. 9, Special Issue 1, pp. 81-87, 2016.

Selected papers from the $7^{\text {th }}$ International Exergy, Energy

and Environment Symposium, IEEE7-2015

Available online at www.jafmonline.net, ISSN 1735-3572, EISSN 1735-3645.

DOI: $10.36884 /$ jafm.9.SI1.25802

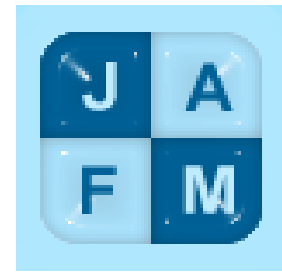

\title{
On the Influence of Low-power Laser Source on the Evaporation of Single Droplets: Experimental and Numerical Approaches
}

\author{
M. H. Sadafi ${ }^{1 \dagger}$, S. González Ruiz², M. R. Vetrano², J. van Beeck², I. Jahn ${ }^{1}$, J. M. Buchlin² \\ and K. Hooman ${ }^{1}$ \\ ${ }^{I}$ School of Mechanical and Mining Engineering, The University of Queensland, St. Lucia, Queensland, 4072, \\ Australia \\ ${ }^{2}$ von Karman Institute for Fluid Dynamics, Chaussee de Waterloo 72, Rhode Saint Gene `se, 1640, Belgium
}

†Corresponding Author Email:m.sadafi@uq.edu.au

(Received October 20, 2015; accepted December 10, 2015)

\begin{abstract}
This work investigates the influence of laser power on an evaporating single droplet made from an $\mathrm{H} 2 \mathrm{O}$ and $\mathrm{NaCl}$ mixture. Heat and mass transfer of a single droplet with the presence of a low-power laser source (as $\mathrm{He}-\mathrm{Ne}$ laser) is studied both numerically and experimentally in this article. A new model is presented to simulate water droplet evaporation. The model is robust enough to be applied for various initial concentrations and conditions of the droplet, ambient conditions, and dissolved media properties. Moreover, laser energy is taken into consideration as a source term which is a function of the wave length of the source beam and refractive index of the droplet. Considering the involved parameters, the model is implemented in a MATLAB code and validated using experimental data obtained in this study on top of those already available in the literature. Experimental data were collected for droplets with an initial radius of $500 \mu \mathrm{m}$ at room temperature for three initial concentrations of $3 \%, 5 \%$, and $10 \%$ (by mass) of $\mathrm{NaCl}$ in water as well as pure water droplet to provide a comprehensive validation dataset. It is shown that low-power laser source significantly increases the evaporation rate $(2.7$ to 5.64 for $0 \%$ and $10 \%$ initial concentration of salt, respectively) which must be taken into consideration while using laser based measurement techniques.
\end{abstract}

Keywords: Saline water; evaporation; laser source; single droplet; heat and mass transfer.

NOMENCLATURE

\begin{tabular}{llll}
$A$ & surface area & $T$ & temperature \\
$c_{p}$ & specific heat & $V$ & volume \\
$D_{v}$ & vapour diffusion coefficient & \multicolumn{2}{c}{ Greek letters } \\
$h$ & heat transfer coefficient & $\alpha$ & attenuation coefficient \\
$h_{D}$ & mass transfer coefficient & $\beta$ & empirical factor \\
$H_{\text {evap }}$ & specific enthalpy of evaporation & $\varepsilon$ & emissivity \\
$k$ & thermal conductivity & $\xi$ & porosity \\
$L$ & length & $\rho$ & density \\
$m$ & area coefficient of infinite fine & \multicolumn{2}{c}{ Subscripts } \\
$\dot{m}$ & mass flow rate & 0 & initial condition \\
$M w$ & molar mass & $c r$ & crust \\
$N u$ & Nusselt number & $C o n$ & conduction \\
$p$ & pressure & $d$ & droplet \\
$p e$ & perimeter & $g$ & gas \\
$P$ & power & $L$ & laser \\
$r$ & radius & $N$ & needle \\
$P r$ & Prandtl number & $S$ & solid \\
$R e$ & Reynolds number & $s t$ & steel \\
$S$ & source term & $v$ & vapour
\end{tabular}




$\begin{array}{ll}S c & \text { Schmidt number } \\ S e & \text { Sherwood number } \\ t & \text { time }\end{array}$

\section{INTRODUCTION}

Laser based measurement techniques are widely used in the field of particle sizing, thermometry, and velocimetry.

Standard rainbow thermometry (SRT) is a laser scattering method which is used to measure the refractive index (hence temperature) and diameter of a single transparent particle (Vetrano et al., 2005). As another example, glory is an interference phenomenon produced due to backscattering of laser light by water droplets (Ruiz et al., 2014).

However, the absorption of laser energy also alters the evaporation process. This study aims to quantify and predict this effect.

To simulate the evaporation process from a solid containing droplet, researchers have suggested two (Abuaf and Staub, 1985; Elperin and Krasovitov, 1995) and three-stage models (Dalmaz et al., 2007; Farid, 2003; Mezhericher et al., 2007; Nešić and Vodnik, 1991). In general, the evaporation of droplets can be described as follows (see Fig.1):

1.The droplet is warmed/cooled to be close to ambient conditions,

2. Evaporation takes place during an isothermal phase,

3. First particles form at the bottom of the droplet (Charlesworth and Marshall, 1960) and grow to cover the upper part,

4.The remaining water is then evaporated followed by complete drying.

The slow formation of the crust is considered in the mathematical modelling by Sadafi et al. (Sadafi et al., 2014b). In their four-stage model they showed that the droplets shrink even after presence of solid particles on the surface which results in a better agreement with the experimental results compared to former models (Sadafi et al., 2014b).

Handscomb et al. (2009) described a generalized model with different possible sub-models for evolution of the droplets following shell formation which includes slow boiling after dry shell formation (Handscomb et al., 2009). They demonstrated that morphology of droplet plays a key role in the droplet evolution after shell formation. Under certain circumstances, a further isothermal stage may exist after shell formation.

Recent experimental studies on a single droplet have been performed using thin filaments to hold the droplet. Temperature measurements of the droplet were performed using two thermocouples in the experimental study by Qi Lin and Chen (Qi Lin and Chen, 2002). Those authors measured the diameter and weight of skim milk droplets to show that droplets could remain attached to the filament $\begin{array}{ll}w c & \text { wet core } \\ \infty & \text { free stream }\end{array}$

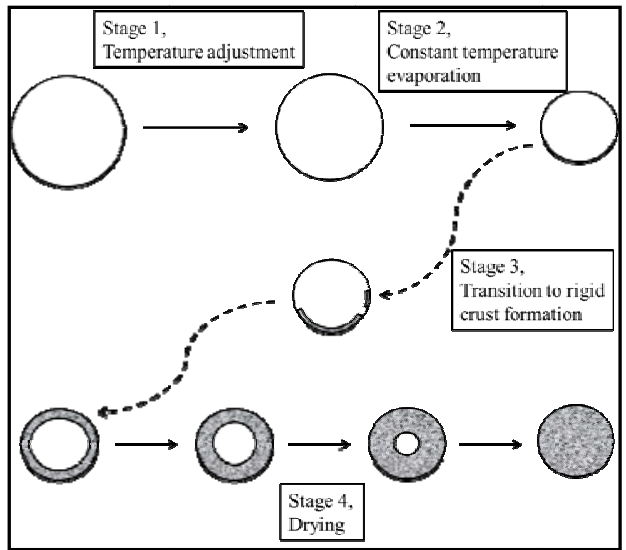

Fig.1. Stage of evaporation of a solid containing water droplet (Sadafi et al., 2014a)

knobs even after the formation of solid parts (Qi Lin and Chen, 2002). Chew et al. used a heated glass filament which was also used as a thermocouple to measure the (high solid concentration milk) droplets temperature (Chew et al., 2013). Using a microscope digital camera, Sadafi et al. monitored the droplet size at low air velocities to note that the droplets shrink after crust formation in slow evaporation under standard room conditions (Sadafiet al., 2014b). In a subsequent study, Sadafiet al. (Sadafiet al., 2015a) monitored the saline water droplet size at different ambient conditions using microscope digital camera. They showed that for $500 \mu \mathrm{m}$ radius droplets with $3 \%$ and $5 \%$ initial $\mathrm{NaCl}$ mass concentrations the net energy required to evaporate the droplet falls by $7.3 \%$ and $12.2 \%$, respectively (compared to a pure water droplet). Also, compared to the time of evaporation of a pure water droplet, the period with wet surface is shorter as a result of crust formation around the saline water droplet. This allows a shorter distance between spray nozzles and heat exchangers (Sadafi et al., 2015a, Sadafi et al., 2016).

In this work, the effect of laser on the evaporation of a suspended droplet is investigated both numerically and experimentally. The output of this work helps researchers to determine the energy absorbed by a single droplet while using laser based measurement techniques.

\section{MATHEMATICAL MODELLING}

The four-stage model developed by Sadafi et al. (Sadafi et al., 2014b, 2015a) was used in this study and is briefly described in this section. It should be mentioned that as this paper focuses on cooling tower conditions, the conditions above the boiling points and fracture of the porous shell due to pressure rise of the gas-vapour mixture are beyond the scope of the current study. However, increase of saturated pressure due to temperature rise is being 
taken into consideration.

According to this model, during the first stage of evaporation, the temperature of a saline water droplet adjusts to approach the wet-bulb temperature. Evaporation in this stage is negligible compared to the later stages. Next, the second stage starts and the droplet size decreases. This stage is an approximately isothermal process with a nearly constant radius reduction and drying rate (Handscomb et al., 2009). As water evaporation continues, solid concentration rises to the critical concentration which, in turn, depends on the solid properties $(27.47 \%$ by mass for $\mathrm{NaCl}$ in water (Bharmoria et al., 2012)). Once the critical concentration is reached, the third stage kicks off and solid crystals are formed in the lower part of the droplet. Then, they start to extend up to the droplet sides as the third stage progresses. Finally, the fourth stage starts once a solid crust forms around the entire droplet. This solid crust then grows in thickness until almost all the liquid is evaporated. This crust is assumed to be a porous medium allowing the diffusion of water vapour through its pores, behaving equivalent to Stefan tubes (Mezhericher et al., 2007).

Fig.2 shows the formed pores on the main crystals. The average diameter of these pores is $1 \mu \mathrm{m}$. During the drying progress, the water mass fraction decreases and the water is replaced by air and vapour in the pores.

Assuming axisymmetric shape for the droplet, homogenous properties (in the temperature adjusting and isothermal evaporation stages), and neglecting directional forces (e.g. gravity) (Ranz and Marshall, 1952), the energy equation for the droplet is (Mezhericher et al., 2007):

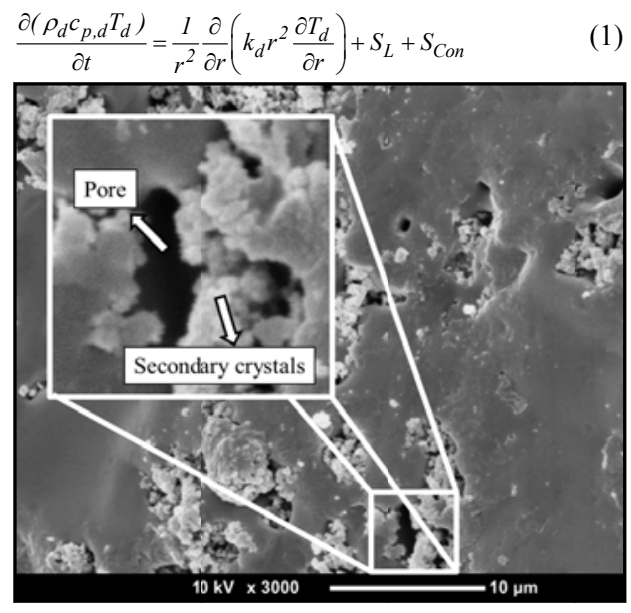

Fig. 2. Close-up image of pores on $\mathrm{NaCl}$ crystal surface using scanning electron microscopy (SEM). Secondary NaCl crystals are created by vapour solidifying at pores (Sadafi et al., 2015b).

where $T$ is the temperature (of the droplet in the first and second stages or of the crust or wet-core during the fourth stage). The source terms due to laser energy (SL) determined as follows:

$$
S_{L}=S_{L, d}+S_{L, N}(2)
$$

Where $S_{L, d}$ and $S_{L, N}$ are the source terms due to the laser power absorbed by the droplet and Needle per volume of the droplet, respectively.

From Beer-Lambert law the absorbed energy is given as (Ingle and Crouch, 1988):

$$
S_{L, d}=P_{L, d}\left(1-e^{\left(-\alpha_{d} D_{d}\right)}\right) / V_{d}
$$

Here, $\alpha_{d}$ is attenuation coefficient which is a function of salt concentration as well as the laser beam wave length. For $\mathrm{NaCl}$ solutions, the corresponding attenuation coefficient was measured experimentally as part of the current study. Based on these the source term for the model can be evaluated.

In a case with a suspended droplet using a needle, considering a Gaussian profile for the laser beam, the hitting of laser beam to the droplet is shown in Fig. 3. If the laser beam spot size is larger than the droplet size, a part of the laser hits the needle as well which is taken into consideration in this work. The source terms due to laser beam hitting the needle $\left(S_{L, N}\right)$ is:

$$
S_{L, N}=\varepsilon P_{L, N} / V_{d}
$$

where $\mathrm{e}_{\varepsilon}$ is the emissivity of the needle which according to Kirchhoff's law is equal to the coefficient of absorption (Chandrasekhar, 1960).

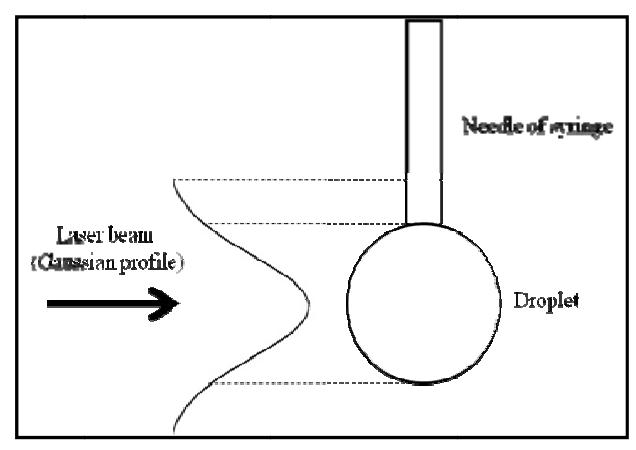

Fig. 3. Hitting of laser beam to the droplet and the needle.

By assuming the needle as an infinite fin the source terms due to conduction through the needle (SCon) is (Incropera, 2007):

$S_{\text {Con }}=\left\{\sqrt{h p e k_{N} A_{N}}\left(T_{g}-T_{N}\right) \tanh \left(m L_{N}\right)\right\} / V_{d}$

where $m^{2}=\frac{h p e}{k_{N} A_{N}}$. Eq. (5) is valid when:

$\tanh \left(m L_{N}\right) \approx 1$

After crust formation in the fourth stage, the solid crystal acts as an insulator between the needle and the wet-core. Therefore, the source terms of $S_{C o n}$ and $S_{L, N}$ are neglected in this stage. Also, due to the same effect of the crust, the effect of laser power in 
the evaporation of the wet-core $\left(S_{L, d}\right)$ is neglected in the fourth stage.

The following boundary conditions are applied for the first and second stage in Eq. (1):

$$
\left\{\begin{array}{lc}
\frac{\partial T}{\partial r}=0 & r=0 \\
h\left(T_{g}-T\right)=k_{d} \frac{\partial T}{\partial r}+H_{\text {evap }} \frac{\dot{m}_{v}}{A_{d}} & r=r_{d}
\end{array}\right.
$$

where $r_{d}$ is the droplet outer radius in the first and second stage. The following conditions apply for the fourth stage:

$$
\left\{\begin{array}{lrl}
\frac{\partial T_{w c}}{\partial r}=0 & r & =0 \\
k_{c r} \frac{\partial T_{c r}}{\partial r}=k_{w c} \frac{\partial T_{w c}}{\partial r} H_{\text {evap }} \frac{\dot{m}_{v}}{A_{c r}} & r=r_{\text {interface }} \\
T_{w c}=T_{c r} & r=r_{\text {interface }} \\
h\left(T_{g}-T_{c r}\right)=k_{c r} \frac{\partial T_{c r}}{\partial r} & r=R_{c r}
\end{array}\right.
$$

where $r_{\text {interface }}$ is the radius of the interface between the wet-core and the crust and $r_{c r}$ is the outer radius of the crust. The evaporating liquid diffuses through the pores to allow the evaporation. As the latent heat is dominant compared to sensible heat (only in isothermal evaporation stage and for the wet-core in the fourth stage), the energy balance equation applied to the first and second stages and the wetcore in the fourth stage can be simplified to (Mezhericher et al., 2007):

$$
H_{\text {evap }} \dot{m}_{v}=h\left(T_{g}-T\right) A_{d}
$$

where $A_{d}$ in the fourth stage is the area of a sphere radius of which is equal to that of the crust (constant). Changes in the droplet radius in the first two stages and the wet-core radius in the fourth stage are determined by solving Eq. (10) (Mezhericher et al., 2007).

$\left(4 \pi r^{2}\right) \frac{d r}{d t}=-\frac{\dot{m}_{v}}{\rho}$

To calculate the wet-core radius in the fourth stage, the left hand side of Eq. (10) is multiplied by the porosity resulting in:

$\left(4 \pi r^{2}\right) \xi \frac{d r}{d t}=-\frac{\dot{m}_{v}}{\rho}$

The vapour mass flow rate from the wet-core in the fourth stage is obtained using Eq. (12) as described in (Sadafi et al., 2014b):

$$
\begin{aligned}
\dot{m}_{v} & =-\frac{p_{g}}{\mathfrak{R}\left(T_{c r, s}+T_{w c, s}\right)} M_{w} D_{v} \frac{8 \pi \xi^{\beta}\left(r_{p} r_{w c}\right)}{\left(r_{c r}-r_{w c}\right)} \\
& \times \ln \left[\frac{p_{g}-p_{v}}{p_{g}-\left(\frac{\Re}{4 \pi M_{w} h_{D} r_{c r}^{2}} \dot{m}_{v}+\frac{p_{v, \infty}}{T_{g}}\right) T_{c r, s}}\right]
\end{aligned}
$$

where $p_{v}$ is the vapour pressure at the interface of the crust and the wet-core and $D_{v}$ is vapour diffusion coefficient. Here, it is assumed that the vapour diffuses through a number of Stefan tubes. The argument in the logarithmic term shows the vapour mass concentration gradient through the pore. The multiplier is the density of the vapour times the vapour diffusion coefficient and the pore cross-sectional area. Besides, $\beta$ is an empirical factor which depends on the structure of the crust. It should be mentioned that for simplification in calculation of mass flow rate in the fourth stage the steady state equation is used.

To calculate the heat and mass transfer coefficients at the gas-liquid interface when gas is forced to flow over (and around) the droplet (during the first and second stages), the correlations from Ranz and Marshall (Ranz and Marshall, 1952) are used:

$$
\begin{aligned}
& N u=2+0.6 \operatorname{Re}_{d}^{\frac{1}{2}} \operatorname{Pr}^{\frac{1}{3}} \\
& S h=2+0.6 \operatorname{Re}_{d}^{\frac{1}{2}} S c^{\frac{1}{3}}
\end{aligned}
$$

By adding a weighting factor " $z$ " the equations of the evaporation and drying (second and fourth) stages are blended (Sadafi et al., 2015a).

A Lagrangian formulation of the flow field is used to solve the governing equations for the first and second stages. A grid with 64 cells in the radial direction is implemented and a fixed time step of 0.1 sis used for the transient implicit solution. For the fourth stage, a uniform grid distribution with size of $4 \mu \mathrm{m}$ in the radial direction is implemented and an adaptive time step with initial value of $0.05 \mathrm{~s}$ is used to obtain the transient implicit solution. Results obtained using shorter (half) time steps and spatially-refined grids are found to be within $1 \%$ of those obtained from the baseline. The obtained results from the model without source terms are validated in (Sadafi et al., 2014b, 2015a).

\section{EXPERIMENTAL STUDY}

\subsection{Experimental Test Rig}

The experimental apparatus as well as the processing routines to optically measure droplet evaporation are presented in Fig. 4. The test rig was installed on an optical table. A $35 \mathrm{~mW}$ laser source with the wave length of $632.8 \mathrm{~nm}$ was used to produce the inlet energy source. The laser beam diameter was $1.23 \mathrm{~mm}$. The laser beam reached the droplet after hitting a 90 degree mirror. The droplet was suspended using a syringe with the diameter of $0.33 \mathrm{~mm}$. A digital camera with a sensor of $1392 \times$ 1024 pixels was used to monitor the size change of the droplet using shadowgraph method. The maximum uncertainty of the image processing was less than $3.5 \%$.

\subsection{Attenuation Coefficient of the Solution}

To measure the attenuation coefficient of $\mathrm{NaCl}$ water different solutions with a range of mass concentrations of salt were prepared. Then, the samples were exposed to a known laser beam. Throughout the experiment, the laser attenuation is measured using a detector placed behind the 


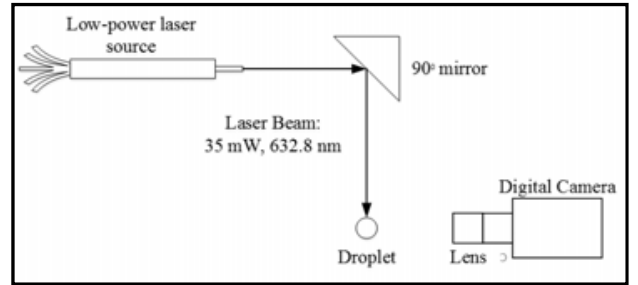

Fig. 4. Test rig; low-power laser source, $90^{\circ}$ mirror, lens, digital camera, and droplet.

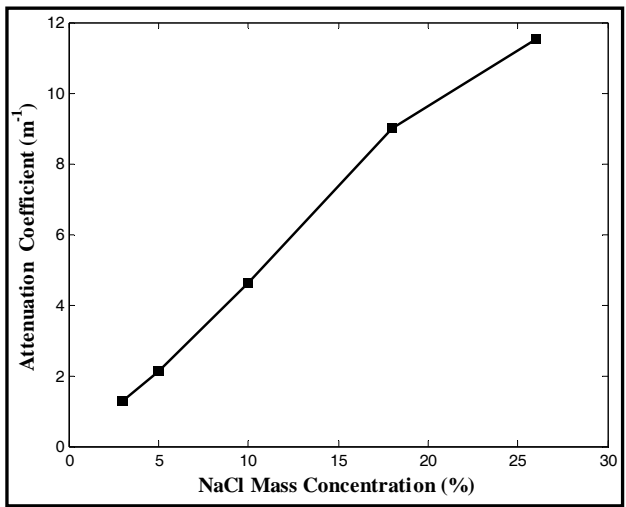

Fig. 5. Influence of $\mathrm{NaCl}$ concentration on the attenuation coefficient of the solution.

droplet. This allows a direct measurement of the attenuation coefficient and its variation as the droplet concentration and size evolves. The obtained results are shown in Fig. 5.

\subsection{Experimental Results}

To investigate the influence of laser energy on the evaporation from an evaporating droplet, different $\mathrm{NaCl}$ concentrations of $0 \%, 3 \%, 5 \%$, and $10 \%$ were tested in the room temperature. The corresponding results are shown in Fig. (6) to Fig. (9), respectively.

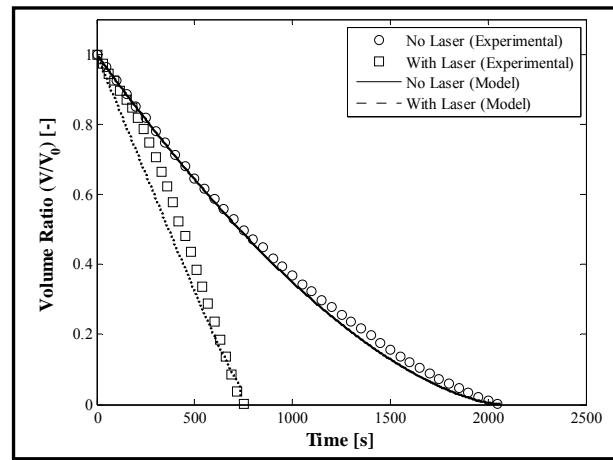

Fig. 6. Influence of laser power on evaporation rate of a pure water droplet at room temperature.

Fig. 6 shows the volume ratio of a single pure water droplet versus time using laser as well as laser free evaporation. As shown in this fig, in both cases the predicted values and experimental results show a good agreement during the whole process. In the case of use of laser source, the experimental results show a considerable change in evaporation time compared to the laser free evaporation which is due to the absorbed external energy by the droplet. The evaporation time ratio for the pure water is 2.71 . This ratio is defined as the evaporation time for a single droplet without laser divided by the evaporation time using a laser beam.

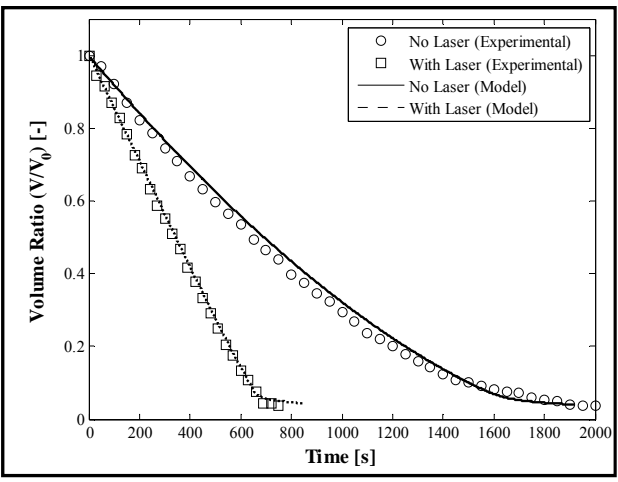

Fig. 7. Influence of laser power on evaporation rate of a $3 \% \mathrm{NaCl}$-water solution droplet at room temperature.

Fig. 7 shows the volume ration of the droplets with $3 \%$ initial concentrations. Sadafi et al. (Sadafi et al., 2015a) presented a criterion to distinguish between the different stages of evaporation based on experimental results using $\mathrm{d} 2$ law. Using this criterion and considering the experimental results without laser source, the beginning of the transient stage is at $805 \mathrm{~s}$ which is in a good agreement with the numerical value of $818 \mathrm{~s}$. Also, the end of transient stage (start of drying stage) for experimental and theoretical results is $1950 \mathrm{~s}$ and $1802 \mathrm{~s}$, respectively.

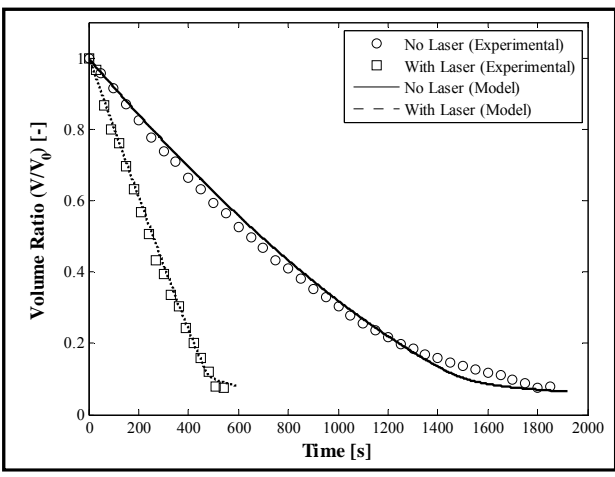

Fig. 8. Influence of laser power on evaporation rate of a $5 \% \mathrm{NaCl}$-water solution droplet at room temperature.

It is shown in Fig. 7 that using saline water droplet increases the time ratio. Here, the initial concentration of the saline water is $3 \%$ by mass. As the initial $\mathrm{NaCl}$ concentration increases, the energy absorbed by the droplet rises and consequently the evaporation rate increases and the drying time is 
shortened. This change in the evaporation time is shown in Figs. 8 and 9. The reason for the increased energy absorbed by the droplet is the influence of $\mathrm{NaCl}$ concentration on the refractive index of the droplet which changes the attenuation coefficient of $\mathrm{NaCl}$-water solutions ( $\alpha_{d}$ in Eq. (3)).

According to Fig. 6 to Fig. 9 and Tab. 1, using laser power to study the evaporation of a single droplet results in a faster evaporation rate compared to laser free evaporation. This is because the evaporation rate in presence of laser beam highly depends on attenuation coefficient of $\mathrm{NaCl}$-water solutions.

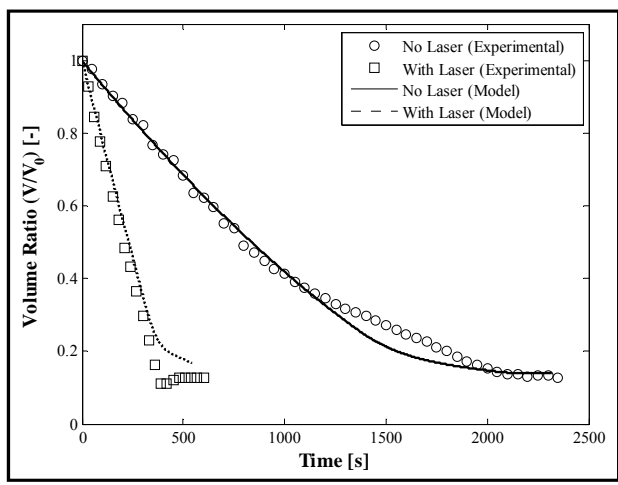

Fig.9. Influence of laser power on evaporation rate of a $10 \% \mathrm{NaCl}$-water solution droplet at room temperature.

For example in the case of the $3 \%$ droplet, the coefficient $\alpha_{d}$ increases from $1.28 \mathrm{~m}^{-1}$ to $8.02 \mathrm{~m}^{-1}$ (at $0 \mathrm{~s}$ and $528 \mathrm{~s}$, respectively), leading to an increase in absorbed energy from $5.57 \times 10^{-5} \mathrm{~W}$ to $8.29 \times 10^{-5} \mathrm{~W}$.

Table 1 compares the evaporation time ratio for pure water droplet, $3 \%, 5 \%$, and $10 \% \mathrm{NaCl}$ initial concentration. For saline water, this ratio is defined as the time of solid particles formation (beginning of the third stage) for a single droplet without laser divided by this time using a laser beam.

Table 1Evaporation time ratio for different initial $\mathrm{NaCl}$ concentrations.

\begin{tabular}{|c|c|c|}
\hline Single droplet & $\begin{array}{c}\text { Initial radius } \\
(\mu \mathrm{m})\end{array}$ & $\begin{array}{c}\text { Evaporation } \\
\text { time ratio }(-)\end{array}$ \\
\hline Pure water & 627.8 & 2.73 \\
\hline $\begin{array}{c}3 \% \text { initial } \\
\text { concentration }\end{array}$ & 494.5 & 2.8 \\
\hline $\begin{array}{c}5 \% \text { initial } \\
\text { concentration }\end{array}$ & 623.5 & 3.52 \\
\hline $\begin{array}{c}10 \% \text { initial } \\
\text { concentration }\end{array}$ & 516 & 5.64 \\
\hline
\end{tabular}

As the concentration rises by time, this coefficient increase which compensates the lower evaporation rate occurring after crust formation (stages three and four). Thus, there is a uniform evaporation rate while using laser power.

\section{CONCLUSION}

A robust model is presented to simulate the heat and mass transfer of a single droplet exposed to lowpower laser source. Laser power is considered as a source term, which results in a significant increase in the evaporation rate. Experimental results obtained in this study, validate the described model. It is shown that as initial salt concentration increases, the evaporation rate rises (drying time decreases). This is due to increase in attenuation coefficient of NaCl-water solutions. Therefore, the energy absorbed while using low-power laser sources in a laser based optic measurement technique must be taken into consideration. The developed model allows a correction to be performed to account for the absorbed energy.

\section{ACKNOWLEDGEMENTS}

This research was performed as part of the Australian SolarThermal Research Initiative (ASTRI), a project supported by the Australian Government, through the Australian Renewable Energy Agency (ARENA) and was funded by the Queensland Geothermal Energy Centre of Excellence (QGECE) Travel Fellowship. The warm hospitality of the von Karman Institute for Fluid Dynamics in Belgium, during this travel fellowship is highly appreciated.

\section{REFERENCES}

Abuaf, N. and F. W.Staub. (1985). Drying of Liquid-solid Slurry Droplets: General Electric, Corporate Research and Development.

Bharmoria, P., H. Gupta, V. P., Mohandas, P. K., Ghosh and A.Kumar. (2012). Temperature Invariance of $\mathrm{NaCl}$ Solubility in Water: Inferences from Salt-Water Cluster Behavior of $\mathrm{NaCl}, \mathrm{KCl}$, and $\mathrm{NH} 4 \mathrm{Cl}$. The Journal of Physical Chemistry B, 116(38), 11712-11719.

Chandrasekhar, S. (1960). Radiative transfer: Courier Dover Publications.

Charlesworth, D. H., and W. R. Marshall. (1960). Evaporation from drops containing dissolved solids.AIChE Journal, 6(1), 9-23.

Chew, J., N. Fu, M. Woo, K. Patel, C. Selomulya, and X. D. Chen. (2013). Capturing the effect of initial concentrations on the drying kinetics of high solids milk using reaction engineering approach. Dairy Science \& Technology, 1-16.

Dalmaz, N., H. O. Ozbelge, A. N. Eraslan, and Y. Uludag. (2007). Heat and Mass Transfer Mechanisms in Drying of a Suspension Droplet: A New Computational Model. Drying Technology, 25(2), 391-400.

Elperin, T. and B. Krasovitov (1995).Evaporation of liquid droplets containing small solid particles.International Journal of Heat and Mass Transfer, 38(12), 2259-2267. 
M. H. Sadafi et al. /JAFM, Vol. 9, Special Issue 1, pp. 81-87, 2016.

Farid, M. (2003).A new approach to modelling of single droplet drying.Chemical Engineering Science, 58(13), 2985-2993.

Handscomb, C. S., M. Kraft, and A. E. Bayly.(2009). A new model for the drying of droplets containing suspended solids.Chemical Engineering Science, 64(4), 628-637.

Incropera, F. P. (2007). Fundamentals of heat and mass transfer. Hoboken, NJ: John Wiley.

Ingle, J. and S. Crouch. (1988). Spectrochemical measurements.Spectrochemical Analysis.

Mezhericher, M., A. Levy, and I. Borde.(2007). Theoretical Drying Model of Single Droplets Containing Insoluble or Dissolved Solids. Drying Technology, 25(6), 1025-1032.

Nešić, S. and J. Vodnik (1991). Kinetics of droplet evaporation.Chemical Engineering Science, 46(2), 527-537.

Qi Lin, S. X. and X. D. Chen. (2002). Improving the Glass-Filament Method for Accurate Measurement of Drying Kinetics of Liquid Droplets. Chemical Engineering Research and Design, 80(4), 401-410.

Ranz, W. E. and W. R. Marshall.(1952). Evaporation from Drops.Chemical Engineering Progress, 48(3), 141-146.

Ruiz, S. G., M. R. Vetrano and J. van Beeck.(2014). Feasibility of using glory and speckle patterns for sizing spherical and irregular particles. Applied Optics, 53(21), 4722-4728.
Sadafi, M. H., I. Jahn, A. B. Stilgoe, and K. Hooman. (2014a). An investigation of evaporation from single saline water droplets: experimental and theoretical approaches. 19th Australasian Fluid Mechanics Conference, Melbourne, VIC.

Sadafi, M. H., I. Jahn, A. B. Stilgoe, A. B. andK. Hooman.(2014b). Theoretical and experimental studies on a solid containing water droplet.International Journal of Heat and Mass Transfer, 78(1), 25-33.

Sadafi, M. H., I. Jahn, A. B. Stilgoe and K.Hooman. (2015a). A theoretical model with experimental verification for heat and mass transfer of saline water droplets.International Journal of Heat and Mass Transfer, 81, 1-9.

Sadafi, M. H., I. Jahnand K.Hooman. (2015b). Cooling performance of solid containing water for spray assisted dry cooling towers. Energy Conversion and Management, 91, 158-167.

Sadafi, M. H., S. González Ruiz, M. R. Vetrano, I. Jahn, J. van Beeck, J. M. Buchlin, and K. Hooman. (2016). An investigation on spray cooling using saline water with experimental verification. Energy Conversion and Management, 108, 336-347.

Vetrano, M. R., J. P. A. J. van Beeck and M. L. Riethmuller.(2005). Assessment of refractive index gradients by standard rainbow thermometry.

Applied optics, 44(34), 7275-7281. 
